\title{
The use of Harmonic Balance in Wave Concept Iterative Method for Nonlinear Radio Frequency Circuit Simulation
}

\author{
Hicham MEGNAFI, Noureddine BOUKLI-HACENE \\ Telecommunication Laboratory of Tlemcen \\ University of Abou Bakr Belkaid \\ Tlemcen, Algeria
}

\author{
Nathalie RAVUE, Henri BAUDRAND \\ Laboratory of Laplace \\ LAPLACE, University of Toulouse, CNRS \\ Toulouse, France
}

\begin{abstract}
This paper presents the birth of the new hybrid method for the non-linear Radio frequency circuits' simulation. This method is based on the combination of the wave concept iterative procedure (WCIP) and the harmonic balance (HB) for their advantages. It consists also the development of an application based on this method for the simulation of nonlinear planar radio frequency circuits. The simulation of the Radio frequency diode implemented in micro-strip line is done. The validations are obtained by the comparison of the results obtained by our new hybrid method and Harmonic Balance under Advanced Design System (ADS) software.
\end{abstract}

Keywords-WCIP; harmonic balance; nonlinear circuits; planar radio frequency circuits; radio frequency diode

\section{INTRODUCTION}

The Technological advances have enabled to establish an efficient microwave circuit. To analyze and simulate them in nonlinear regime, several methods of analysis have been implemented to achieve the required performances [1], [2], the basic differences between these methods that exist in the domain of analysis are temporal or frequency.

The nonlinear system response is periodic that can be determined by integrating the differential equations that govern the circuit to steady state.

The Time-domain analysis, if it allows to take in consideration a high number of non linearities, then it is not suitable for circuits having relatively large time constant elements in front of the period of the applied signal. In the other hand, if this approach allows the distortions study, it only applies to circuits with low nonlinearities [3].

To remove the main problems caused by the two previous methods, an alternative approach has been proposed and become the most commonly used method in the analysis of nonlinear circuits. This method is called the harmonic balance (HB) [4].

The basic principle of the harmonic balance is to split the circuit into two sub-circuits; a linear and nonlinear sub-circuits. The linear sub-circuit is necessary to be studied in the frequency-domain and the non-linear sub-circuit is well described in the time-domain. The results of the time-domain analysis are then converted into the frequency-domain by the Fourier Transform method [4]. The convergence is obtained, only if the interconnection currents between the linear and nonlinear sub-circuits, for each harmonic, are the same. The different currents must be balanced for each harmonic [5].

In the other hand, another method that has already shown its effectiveness in the simulation of RF circuits is called the wave concept iterative process (WCIP); this method is based on the concept of waves. It's applied in the planar structures of resolution [6], the micro-strip antennas [7] and the planar filters [8].This method has the advantage of simplicity because it does not involve the use of basic functions and matrix inversion as in other calculation methods. Therefore, it is possible to analyze a large complex planar microwave structures [9], [10]. Moreover, a high computational speed can be achieved by using the two dimensional the fast Fourier transformation algorithm known as fast modal of transformation (FMT) [11].

The classical wave concept iterative process was not applied in the simulation of nonlinear circuits because this method is based on frequency domain analysis, for which we proposed a combination of HB and WCIP to solve the problem of limited simulation; HB and WCIP are based on the use of the Fourier transform and its inverse. The balance between the two domains related to the Fourier transform is fundamental to develop a model of nonlinear electromagnetic problems and to qualify circuits in microwaves.

The rest of the paper is organized as follows: The next one (Section II) contains a definition of the wave concept, then we describe the iterative process and its algorithm. We are going to show the interest and the principle of the harmonic balance and its algorithm. In Section III, we propose a new approach of WCIP (hybrid method of HB and WCIP) to get the advantages of the two methods for the simulation of nonlinear circuits, we propose also a nonlinear RF diode modeling in small signal regime to validate this new hybrid approach. Where in Section IV, we present the obtained results validated by the comparison with the results obtained by ADS software. And in the last Section $\mathrm{V}$, we present the perspective of this new hybrid method as an improvement of the convergence and the simulation of new non-linear circuit (such as transistor, nonlinear capacitor) and a conclusion. 


\section{METHODS}

\section{A. Wave Concept Iterative Method}

The main goal of this part is to propose a global electromagnetic analysis for characterizing microwave planar linear circuits based on the use of the iterative method. This method is based on the wave concept. It has the advantage of simplicity because it does not involve the use of basic functions and matrix inversion as in other calculation methods. Therefore, it is capable of analyzing large complex planar microwave structures [12].

\section{1) Waves Definition}

The wave concept is introduced by writing the transverse electric field $\vec{E}_{i}$ and transverse current density $\vec{J}_{1}$ in terms of incident $\overrightarrow{A_{1}}$ and reflected $\vec{B}_{i}$ waves in each medium $i$. It leads to the following set of equations [10], [11]:

$$
\left\{\begin{array}{l}
\vec{A}_{i}=\frac{1}{2 \sqrt{Z_{0}}} \cdot\left(\vec{E}_{i}+Z_{0 i} \cdot \vec{J}_{i}\right) \\
\vec{B}_{i}=\frac{1}{2 \sqrt{Z_{0}}} \cdot\left(\vec{E}_{i}-Z_{0 i} \cdot \vec{J}_{i}\right)
\end{array}\right.
$$

Where, $\mathrm{Z}_{0}$ is an intrinsic impedance of the medium.

\section{B. Principle of Wave Concept Iterative Method}

According to the criterion defining the wave concept, we can define the electric field and the current density values in each point of the surface $\Omega$ by the determination of the incidental and the reflective waves values. So, the iterative process is based on the creation of a recurrence relation between the incidental and the reflective waves, and the repetition of this relation until convergence [9].

The iterative method can be summarized by the following equations system [3], [4]:

$$
\left\{\begin{array}{c}
\vec{A}=\hat{S} \cdot \vec{B}+\vec{A}_{0 i} \\
\vec{B}=\hat{\Gamma} \cdot \vec{A}
\end{array}\right.
$$

Where,

$\hat{S}$ : The transmission operator at the interface $\Omega$,

$\hat{\Gamma}$ : The reflection operator representing the half medium around the interface

$\overrightarrow{\mathrm{A}}_{0 \mathrm{i}}$ : The source wave that can be defined by:

$$
\vec{A}_{0 i}=\frac{\vec{E}_{0}}{\sqrt{Z_{0 i}}}
$$

$\overrightarrow{\mathrm{E}}_{0}$ : The total electric field produced by the excitation source.

The first system equation (2) describes the electromagnetic behavior at the circuit interface in spatial domain, while the second system equation (2) describes the waves' reflection in modal-domain.

The iterative process use successively these two equations through a Fast Modal Transform (FMT: from spatial domain to modal domain) and its inverse (conversion from the modal domain to the spatial one) [13].
Wave propagation described by incident and reflected waves, is presented in the planar structure. We can see that the waves will be reflected continuously, as shown in Fig. 1 [14].

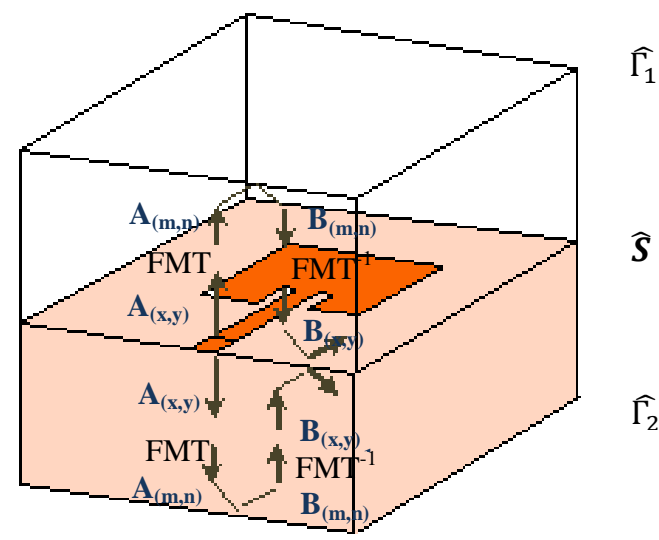

Fig. 1. Wave propagation in planar circuit [5].

\section{1) Wave Concept Iterative Procedure}

The procedure of the wave concept iterative method is

\begin{tabular}{|c|c|}
\hline Steps & Development \\
\hline First step & $\begin{array}{l}\text { Define the excited wave of planar source in spatial- } \\
\text { domain } \overrightarrow{\mathrm{A}_{0}^{1}} \text {. }\end{array}$ \\
\hline second step & $\begin{array}{l}\text { transform the incident waves in the spatial-domain to the } \\
\text { model-domain by the FMT (Fast Modal Transform) : } \\
\overrightarrow{A_{(m, n)}^{l}}=F M T\left(\overrightarrow{A_{(x, y)}^{l}}\right)\end{array}$ \\
\hline Third step & $\begin{array}{l}\text { get the incident waves in model-domain by the application } \\
\text { of the reflection coefficient: } \\
\overrightarrow{B_{(m, n)}^{l}}=[\Gamma] \cdot \overrightarrow{A_{(m, n)}^{l}}\end{array}$ \\
\hline Fourth step & $\begin{array}{l}\text { convert the waves in the model-domain to the spatial- } \\
\text { domain by the FMT inverse: } \\
\overrightarrow{B_{(x, y)}^{l}}=F M T^{-1}\left(\overrightarrow{B_{(m, n)}^{l}}\right)\end{array}$ \\
\hline Fifth step & $\begin{array}{l}\text { Calculate the reflected waves using the scattering } \\
\text { parameters of planar circuit: } \\
\overrightarrow{A_{(x, y)}^{l}}=[S] \cdot \overrightarrow{B_{(x, y)}^{l}}+\overrightarrow{A_{0}^{l}}\end{array}$ \\
\hline Sixth step & $\begin{array}{l}\text { Repeat the second step to the fifth step until the } \\
\text { convergence of the } \mathrm{S} \text { parameters are obtained. }\end{array}$ \\
\hline Seventh step & $\begin{array}{l}\text { After testing the convergence, we can calculate the } \\
\text { tangential electric field and current density }\end{array}$ \\
\hline
\end{tabular}
summarized by the following steps which are shown in Table I:

TABLE I. STEPS of WAVE ConcePt Iterative Process Procedure

\section{Harmonic Balance Method}

The Harmonic balance is a hybrid time and frequency domains analysis technique for simulating nonlinear circuits and systems. This hybrid analysis allows all the advantages of nonlinear temporal domain modeling, combined with the strength (efficiency) of the steady-state frequency technique [5].

\section{1) The Standard Harmonic Balance Technique}

The basic principle of HB approach is to split the circuit into two sub-circuits of $\mathrm{N}$ common ports between the linear and non-linear sub-circuits. Each branch of the circuit circulates a current harmonic component [15], [16]. In this work, the nonlinear sub-circuit consists in a nonlinear diode represented by an algebraic equation $i(t)=F(v(t))$, so the circuit is decomposed as is shown in Fig. 2. 


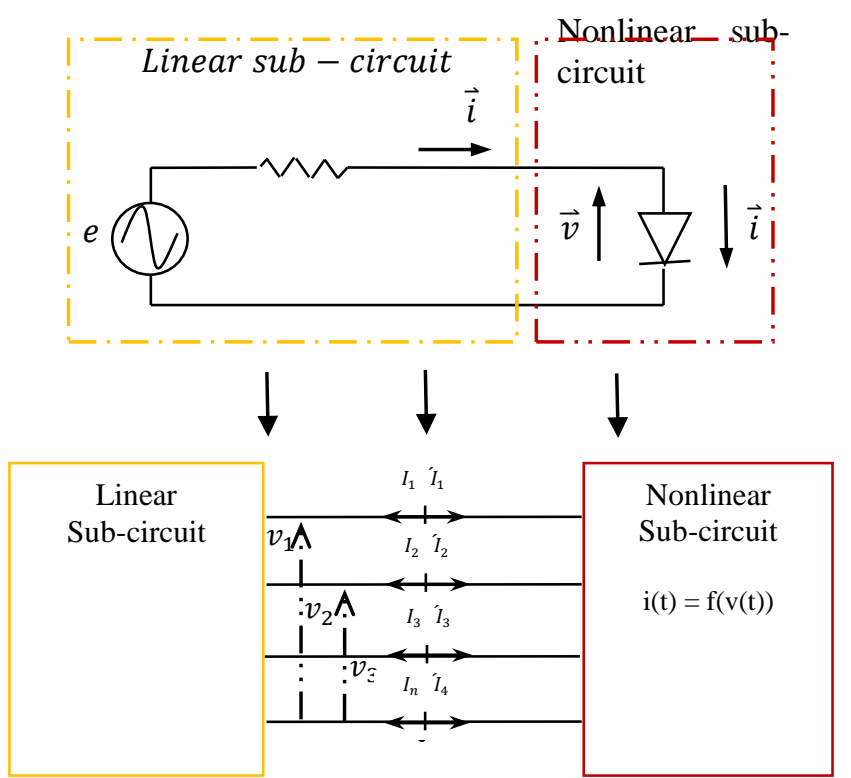

Fig. 2. The Harmonic Balance method split the circuit into linear and nonlinear subcircuits.

The currents flowing from nodes into linear sub-circuit $\widehat{\mathrm{I}_{\mathrm{k}}}$, including all distributed elements, are calculated by means of a frequency-domain linear analysis. And the Currents from nodes into nonlinear sub-circuit $\widehat{\mathrm{I}}_{\mathrm{k}}^{\prime}$ are calculated in the timedomain. The balance between the time domain and frequency domains is obtained by Fourier transform; it is necessary to use a sinusoidal excitation to apply this technique. According to Kirchoff's Current Law (K.C.L), the currents sum should be zero at all nodes (4) [17].

$$
\widehat{I_{k}}\left(k . \omega_{0}\right)+\widehat{I_{k}^{\prime}}=0 \forall k \in[0, \ldots, K]
$$

Where, $\mathrm{K}$ is the number of harmonic, $\omega_{0}$ is the pulsation of the source.

The currents' sum computation gives the error of the method, called $\mathrm{F}_{\text {error }}(5)$. If the method converges (i.e. if the error function is driven to a given small value) then the resulting voltage amplitudes and phases approximate the steady-state solution [17], [18].

$$
F_{\text {error }}=\widehat{I}_{k}(\omega)+\widehat{I}_{k}^{\prime}(\omega) \forall k \in[0, \ldots, K]
$$

Where; $\mathrm{N}$ is the number of harmonic, $\omega$ is the pulsation of harmonic for each node.

\section{2) Algorithm of Harmonic balance}

The procedure of harmonic balance is summarized by the following steps which are shown in Table II:
TABLE II. STEPS OF HARMONIC BALANCE PROCEDURE

\begin{tabular}{|l|l|}
\hline Steps & Development \\
\hline First step & $\begin{array}{l}\text { Initial the estimation of V0(t) (can be also in the } \\
\text { frequency domain), we can estimate the initialization of } \\
\text { the excitation by pulses with a broad spectral content or } \\
\text { by a value equals to zero. }\end{array}$ \\
\hline Second step & $\begin{array}{l}\text { Apply nonlinearity in the time-domain, } \\
\mathrm{i}(\mathrm{t})=\mathrm{F}(\mathrm{v}(\mathrm{t}))\end{array}$ \\
\hline Third step & $\begin{array}{l}\text { Convert the current in the time-domain to the frequency } \\
\text { domain by the FFT. }\end{array}$ \\
\hline Fourth step & $\begin{array}{l}\text { In the frequency-domain, Check if the harmonics are } \\
\text { balanced using the cost, (the use of Kirchoff's Current } \\
\text { Law equation). }\end{array}$ \\
\hline Fifth step & $\begin{array}{l}\text { Still in the frequency-domain, If the convergence criteria } \\
\text { were not satisfied, updated the voltages using the cost } \\
\text { function and its Jacobian. } \\
\mathrm{v}(\mathrm{f})^{\mathrm{k}+1}=\mathrm{v}(\mathrm{f})^{\mathrm{k}}-\mathrm{J}^{-1}\left(\mathrm{v}(\mathrm{f})^{\mathrm{k}}\right)\end{array}$ \\
\hline Sixth step & $\begin{array}{l}\text { Convert the voltage in the frequency-domain to the time- } \\
\text { domain by the FFT-1. }\end{array}$ \\
\hline Seventh step & $\begin{array}{l}\text { Repeat the second step to the sixth step until the } \\
\text { convergence is done. }\end{array}$ \\
\hline
\end{tabular}

The Harmonic Balance algorithm works as it is illustrated in Fig. 3 [19].

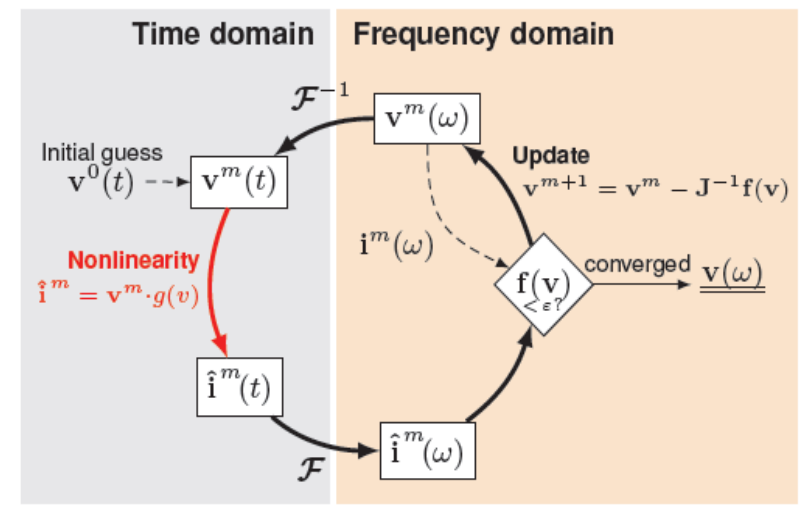

Fig. 3. Algorithme working principle.

\section{NEW HYBRID APPROACH FOR NONLINEAR RADIO FREQUENCY CIRCUIT SIMULATION}

In this part, we present the new approach of WCIP (hybrid HB-WCIP); it is a combination between WCIP classical and HB that we have proposed as well as their algorithm (hybrid HB-WCIP algorithm).

\section{A. Principle}

In the WCIP, a single frequency is used for the linear circuit excitation, so we can say that the wave is defined in space (pixel) and in the frequency at the circuit interface, hence the study is spatial-frequency at the circuit interface. To simulate the non linear circuit, the principle of $\mathrm{HB}$ is used to split the circuit into two sub-circuits at its interface; the linear sub-circuit is simulated by the classical iterative method, while the non-linear sub-circuit is solved by a temporal resolution. In this way, we took advantage of the WCIP in the simulation of the linear part of the circuit, and the principle of the harmonic balance in the simulation of the non-linear part. 


\section{B. Algorithm}

The use of harmonic balance in the wave concept iterative method can be summarized by the diagram illustrated in Fig. 4 .

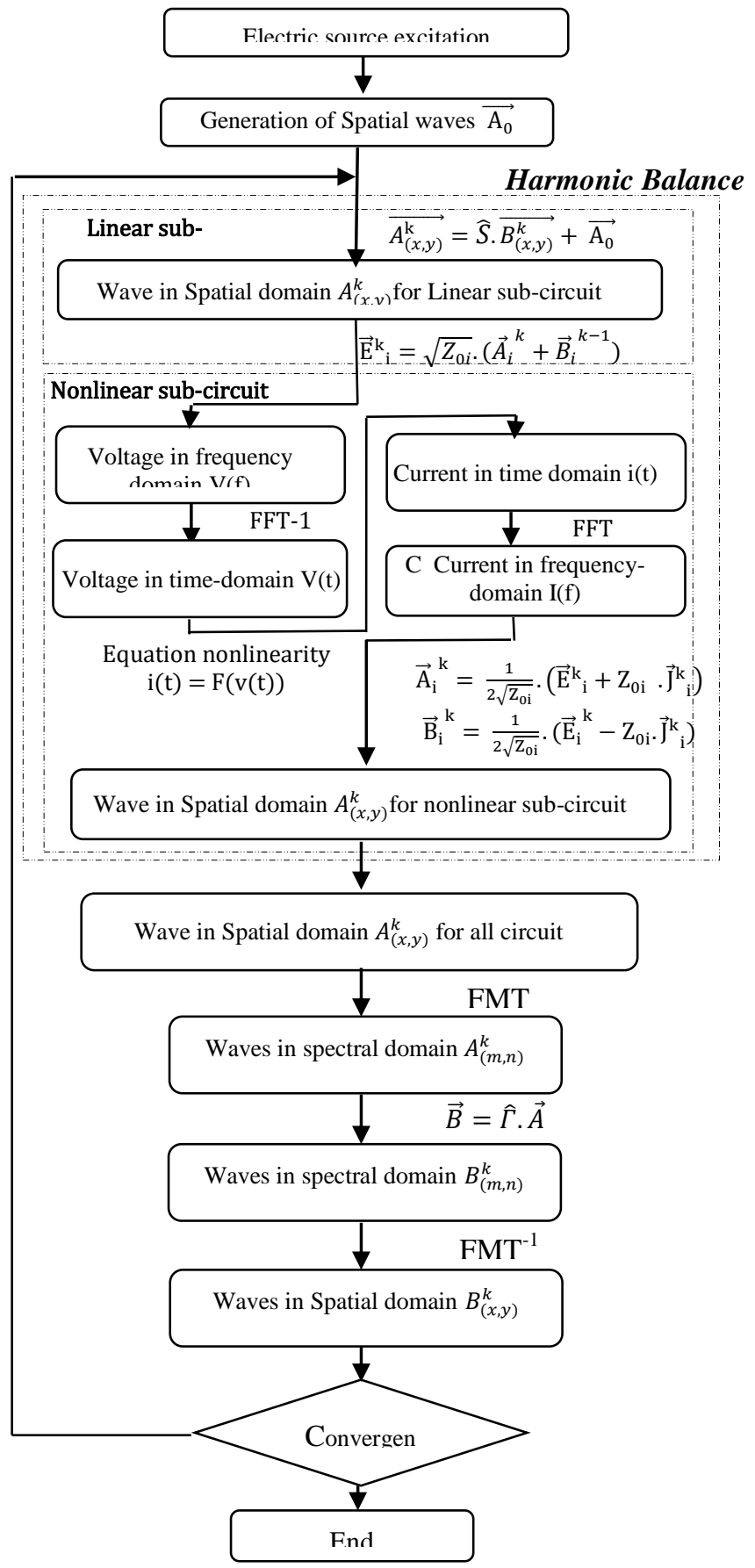

Fig. 4. Harmonic balance - Wavec concept iterative process algorithm.

\section{Nonlinear Radio Frequency Diode Modeling for Small Signal Regime}

As it is noted previously, the modeling of nonlinear subcircuit that we want to simulate is essential. In this part, we take a diode as an example of a nonlinear sub-circuit, and we propose a diode model that functions in the small signal regime.
The characteristic equation of a diode is given by the following formula [20]:

$$
I(V)=I_{S}\left(e^{\alpha . V}-1\right)
$$

With: $\mathrm{V}$ is the Voltage across the diode junction (volts)

$$
\mathrm{I}_{\mathrm{s}} \text { Saturation Current (amperes) }
$$

$$
\alpha=\frac{q}{\eta \cdot \kappa \cdot T}
$$

$\mathrm{q}$ is the electron charge $=1.6^{*} 10^{-19}$ (coulomb),

$\eta$ is diode Ideality Factor (generally between 1.1 and 1.2)

$\kappa$ is Boltzmann's constant $=1.38^{*} 10^{-23}\left(\right.$ joule $\left./{ }^{\circ} \mathrm{K}\right)$,

$\mathrm{T}$ is temperature in degrees Kelvin $\left({ }^{\circ} \mathrm{K}\right)$,

Current flowing through the diode obtained by (6) contains two components, alternative $i(t)$ and continuous $I 0$, for this the total current $\mathrm{I}=\mathrm{I} 0+\mathrm{i}(\mathrm{t})$, the same for the voltage, $\mathrm{V}=\mathrm{V} 0+\mathrm{v}$ $(t)$. Where $v(t)$ is the alternating voltage that consists of $n$ harmonics, hence the formula of the total voltage is as follows:

$$
V(t)=V_{0}+V_{1} \cdot \cos \left(\omega_{0} \cdot t\right)+\cdots V_{n} \cdot \cos \left(n \cdot \omega_{0} \cdot t\right)
$$

Where, $\mathrm{V}_{\mathrm{n}}$ is the $\mathrm{nth}$ harmonic component of the voltage.

In the approximation small signal (i.e.: $\mathrm{i}(\mathrm{t})<<\mathrm{I} 0)$, the diode current can be developed in Taylor's series:

$$
I(V)=I_{0}+\left.\frac{V}{1 !} \cdot \frac{d I}{d V}\right|_{V 0}+\left.\frac{V^{2}}{2 !} \cdot \frac{d^{2} I}{d^{2} V}\right|_{V 0}+\cdots+\left.\frac{d^{n} I}{d^{n} V}\right|_{V 0}
$$

with

$$
\left.\frac{d I}{d V}\right|_{V 0}=G_{d},\left.\frac{d^{2} I}{d V^{2}}\right|_{V 0}=\alpha \cdot G_{d},\left.\frac{d^{3} I}{d V^{3}}\right|_{V 0}=\alpha^{2} \cdot G_{d}
$$

Where, Gd is the dynamic conductance of the diode junction when the two different frequency voltages are applied across the diode.

The formula of current (9) will be:

$$
\mathrm{I}(V)=I_{0}+G_{d} \cdot\left(\frac{V}{1 !}+\alpha \cdot \frac{V^{2}}{2 !}+\alpha^{2} \cdot \frac{V^{3}}{3 !}+. .+\alpha^{n-1} \cdot \frac{V^{n}}{n !}\right)
$$

To simplify the current (9) we use the Pascal triangle formula (11) to solve the current equation.

$$
(a+b)^{n}=\sum_{k=0}^{n} C_{n}^{k} \cdot a^{n-k} \cdot b^{k}
$$

After calculating, the current formula is described as follows:

$$
I(V)=I_{0}+G_{d} \cdot \sum_{k=1}^{n} \frac{\alpha^{k-1}}{k !} \cdot\left[\ldots\left[\sum_{k_{m-1}=0}^{k_{m-2}} C_{k_{m-2}}^{k_{m-1}} \cdot V_{m-1}^{k_{2}-k_{m-1}} \cdot V_{m}^{k_{m-1}}\right]\right]
$$

With $\mathrm{C}_{\mathrm{n}}^{\mathrm{m}}$ is the coefficient obtained by the Pascal triangle, $\mathrm{n}$ is the harmonic number, $\mathrm{V}_{\mathrm{n}}^{\mathrm{m}}$ is the $\mathrm{nth}$ harmonic component of the voltage for $m$ iterations.

So we can implement this formula in the HB-WCIP algorithm, so that the RF diode can be simulated in the nonlinear regime. 


\section{NUMERICAL RESULTS}

The validation of the new approach algorithm of WCIP (hybrid HB-WCIP) can be confirmed by the simulation of a diode RF implemented in micro-strip line.

The RF diode model already proposed in Section $\mathrm{C}$ of Section III, works in the regime of small signals because we have used Taylor's development. Hence the input power of the source must be low enough to keep this regime.

The micro-strip line is deposited on a relative electric substrate permittivity $\varepsilon_{2}=9.6$, thickness $0.635 \mathrm{~mm}$, and width $\mathrm{W}=0.375 \mathrm{~mm}$. The ambient environment is air, therefore the relative electric substrate permittivity $\varepsilon_{1}=1$.

The structure shown in (Fig. 5) is enclosed in a box with electrical walls. The box dimensions and heights of the two medium are described as follows:

$$
\mathrm{h} 1=4 \mathrm{~mm}, \mathrm{~h} 2=0.635 \mathrm{~mm}, \mathrm{a}=6 \mathrm{~mm} \text { et } \mathrm{b}=6 \mathrm{~mm} \text {. }
$$

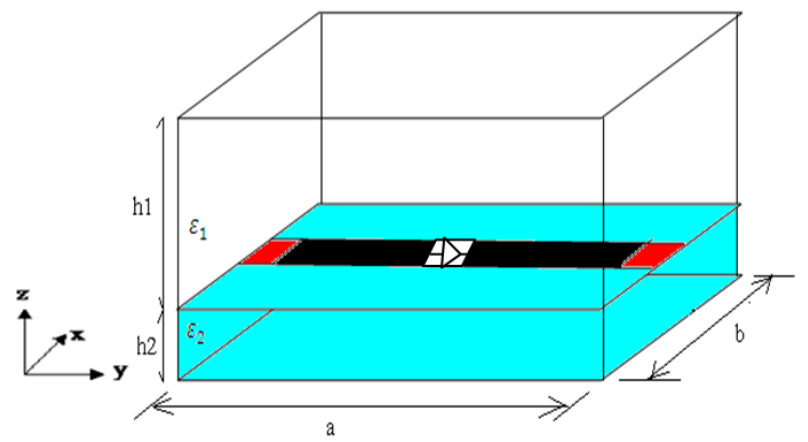

Fig. 5. Radio frequency diode implemented in micro-strip line.

The supply source type of this structure is bilateral and polarized along the axis $\mathrm{Ox}$, and characterized by internal impedance $\mathrm{ZO}$ considered as the paralleling of the characteristic impedances of the two medium in which is delivered, i.e.:

$$
Z_{0}=Z_{01} / / Z_{02}=\frac{Z_{01} \cdot Z_{02}}{Z_{01}+Z_{02}}=90.57 \mathrm{Ohm}
$$

To stay in small signal regime, we set the power of the source at $-20 \mathrm{dbm}$. The frequency of the source is $2 \mathrm{GHz}$.
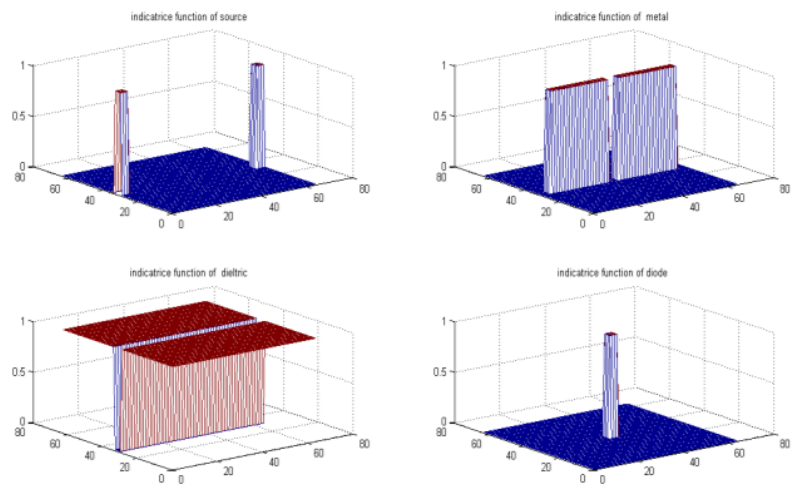

A mesh of $64 \times 64$ pixels has been chosen to approach the real dimensions (for a good description of the electromagnetic field in the micro-strip line, a mesh of 4 to 5 pixels in the width of the line must be established, one or two pixels for each end describe the boundary conditions, and 1 and more pixels in the middle to express its value inside the line).

The indicator functions of each medium (source, metal, dielectric and diode) are shown in the above (Fig. 6):

The characteristics of the RF diode implemented in the line are described as follows:

Saturation Current $\mathrm{I}_{\mathrm{s}}: 5.10-8 \mathrm{~A}$

Diode Ideality Factor $\eta: 1.05$

Temperature $\mathrm{T}: 300^{\circ} \mathrm{K}$

To validate the results obtained by the new algorithm implementation of WCIP (hybrid HB-WCIP) in Matlab, we compared these results with ADS software. The following figure shows the RF diode simulation implemented in the micro-strip line with these features under ADS software (Fig. 7).

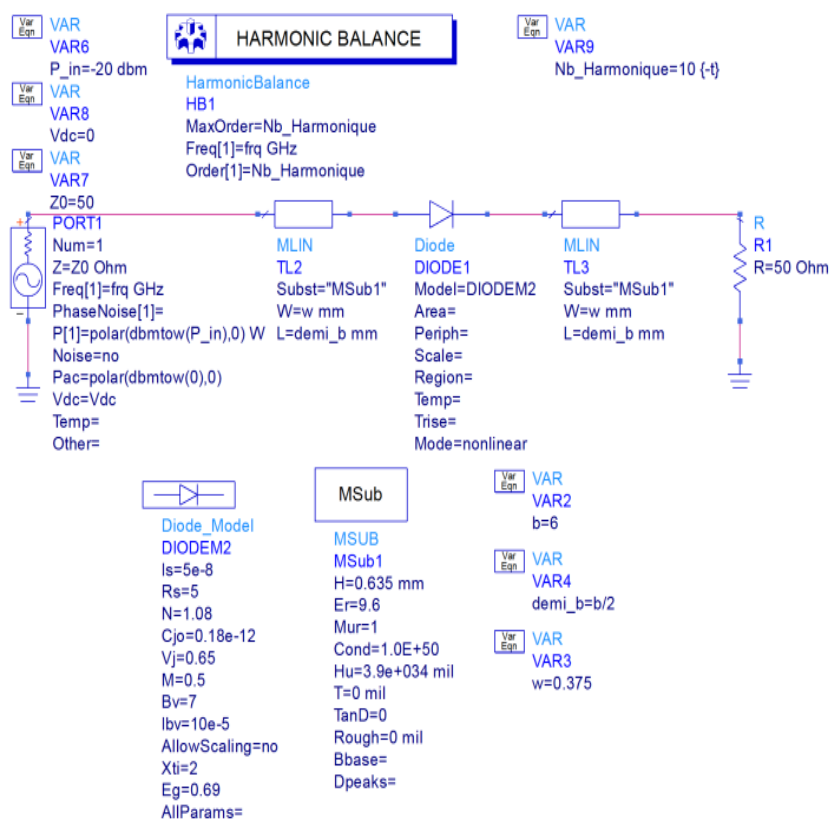

Fig. 7. Simulation by ADS software.

With a maximum harmonic number equals to 5 , the implementation results of the new algorithm (hybrid HBWCIP) as obtained by ADS is described in the following figure (Fig. 8):

Fig. 6. The indicator functions of all mediums. 


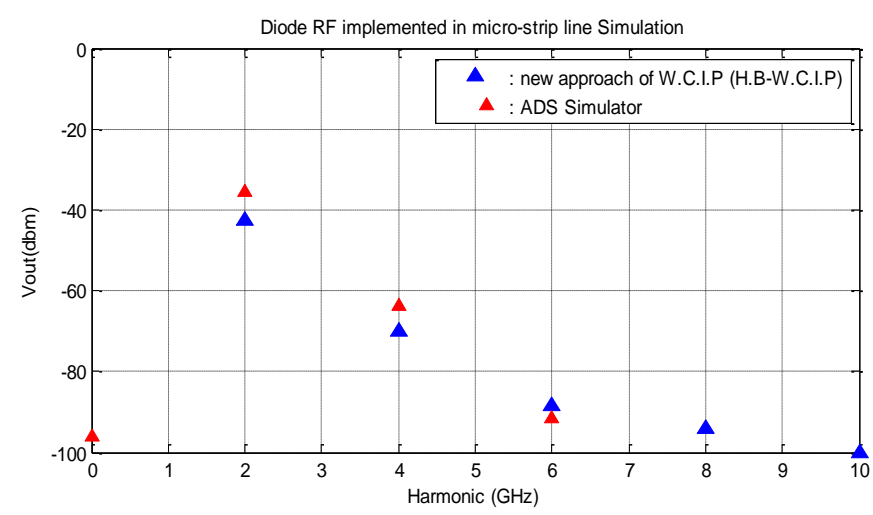

Fig. 8. Radio Frequency diode simulation results implemented in the microstrip line by the change of the harmonic number used equals to 5 .

The figure shows clearly the simulation with the new approach of WCIP (HB-WCIP) and the results obtained by ADS software are comparable, except in the last harmonics (harmonic 4 and 5). This difference comes back to the techniques of analysis used in each method.

A second comparison is needed between the new method (HB-WCIP) and HB implemented under ADS Software to study the convergence of results; the necessary harmonics number to reach a best presentation of the output voltage at the diode. For this, we simulate the RF diode implanted in the micro-strip line by HB.WCIP under Matlab and by ADS software for different number of harmonic.

The following table (Table III) shows the values in dbm of the output voltage harmonics (Fig. 7) obtained by the two methods; HB-WCIP and HB under ADS Softawre, for the number of harmonic used from 1 to 7 .

TABLE III. RF DIODE SIMULATION RESULTS IMPLEMENTED IN THE MiCRO-STRIP LINE BY THE CHANGE OF THE HARMONIC NUMBER USED

\begin{tabular}{|c|c|c|c|c|c|c|c|c|}
\hline \multirow{2}{*}{\multicolumn{2}{|c|}{$\begin{array}{l}\text { Number of } \\
\text { harmonic used } \\
\text { in } \\
\text { each simulation }\end{array}$}} & \multicolumn{7}{|c|}{ Number of harmonic (dbm) } \\
\hline & & \multirow{2}{*}{$\begin{array}{l}\text { Ist } \\
-\end{array}$} & \multirow{2}{*}{$\begin{array}{l}\text { 2rd } \\
\mathrm{X}\end{array}$} & \multirow{2}{*}{$\begin{array}{l}\text { 3rd } \\
\mathrm{X}\end{array}$} & \multirow{2}{*}{$\begin{array}{l}\text { 4th } \\
X \\
\end{array}$} & \multirow{2}{*}{$\begin{array}{l}5 \text { th } \\
X\end{array}$} & \multirow{2}{*}{$\begin{array}{l}\text { 6th } \\
X \\
\end{array}$} & \multirow{2}{*}{$\begin{array}{l}\text { 7th } \\
X\end{array}$} \\
\hline 1 & $A D S$ & & & & & & & \\
\hline 1 & $H B-W C I P$ & - & $\mathrm{X}$ & $\mathrm{X}$ & $\mathrm{X}$ & $\mathrm{X}$ & $\mathrm{X}$ & $\mathrm{X}$ \\
\hline \multirow{2}{*}{2} & $A D S$ & - & - & $\mathrm{X}$ & $\mathrm{X}$ & $X$ & $\mathrm{X}$ & $\mathrm{X}$ \\
\hline & $H B-W C I P$ & - & - & $\mathrm{X}$ & $\mathrm{X}$ & $\mathrm{X}$ & $\mathrm{X}$ & $\mathrm{X}$ \\
\hline \multirow{2}{*}{3} & $A D S$ & - & - & - & $\mathrm{X}$ & $\mathrm{X}$ & $\mathrm{X}$ & $\mathrm{X}$ \\
\hline & $H B-W C I P$ & - & - & -87.6 & $\mathrm{X}$ & $\mathrm{X}$ & $\mathrm{X}$ & $\mathrm{X}$ \\
\hline \multirow{2}{*}{4} & $A D S$ & - & - & - & - & $X$ & $\mathrm{X}$ & $\mathrm{X}$ \\
\hline & $H B-W C I P$ & - & - & - & -96.5 & $\mathrm{X}$ & $\mathrm{X}$ & $\mathrm{X}$ \\
\hline \multirow{2}{*}{5} & $A D S$ & - & - & - & - & - & $\mathrm{X}$ & $\mathrm{X}$ \\
\hline & $H B-W C I P$ & - & - & - & - & - & $\mathrm{X}$ & $\mathrm{X}$ \\
\hline \multirow{2}{*}{6} & $A D S$ & - & - & - & - & - & - & $X$ \\
\hline & $H B-W C I P$ & - & - & - & -94.3 & -96.5 & - & $\mathrm{X}$ \\
\hline \multirow{2}{*}{7} & $A D S$ & - & - & - & - & - & - & - \\
\hline & $H B-W C I P$ & - & - & - & -94.3 & -96.5 & - & - \\
\hline
\end{tabular}

We can consider that the harmonic values of the output voltage less than $-100 \mathrm{dbm}$ are negligible noise.

In Table III, we can see clearly that the harmonic values of the output voltage obtained by ADS software are stable at a maximum harmonic number of three, hence three harmonic is necessary to present the output voltage of the diode. While in the HB-WCIP requires a number of harmonic equals to five. On the other hand, the values of the harmonics of the output voltage obtained by the two methods are really comparable between them.

According to the results obtained by the previous simulations, we can conclude that the simulation results are really comparable with those obtained by HB under ADS software. The small difference between the harmonic number is necessary to reach the convergence returns to the techniques of analysis used in each method, as well as WCIP, taking in consideration the box effect, on the other hand ADS neglects this effect.

\section{CONCLUSION AND FUTURE SCOPE}

In this study, we have started with the presentation of the non-linear circuits' simulation interest, then the advantages and disadvantages of analysis methods of these circuits. After that we have presented the iterative method concept as well as the harmonic balance method. After that, we have proposed a new WICP approach (a hybrid HB-WICP) for the circuit non-linear simulation joined with R.F model diode to validate this approach. The validations are obtained by the comparison of the results obtained by our new hybrid method and HB under ADS software.

Therefore, the future scope of the proposed approach can be developed in the minimization of the time convergence. We can also propose other model of non-linear circuit for small signal regime and also for large signal regime such as transistor, non-linear capacitor.

\section{REFERENCES}

[1] M.B. Stree, "Simulation of nolinear microwave circuit- an historical perspective and comparisons," 1991 IEEE MTT-S Microwave Symp Dig., vol. 2, pp. 599-602, 1991.

[2] J. Kunisch, A. Bahr, M. Rittweger, I. Wolff, "Analysis of nonlinear circuits using the compression approach," Proc. IEEE MTT-S Int. Microwave Symp. Digest, pp. 637-640, 1993.

[3] M. I. Sobhy, E. A. Hosny, M. W. R. Ng, E. A. Bakkar, "Nonlinear system and subsystem modeling in time domain," IEEE Trans. Microw. Theory Tech, vol. 44, no. 12, pp. 2571-2579, Dec. 1998.

[4] A. Mehrotra, A. Somani, "A Robust and Efficient Harmonic Balance (HB) using Direct Solution of HB Jacobian," In Proc. IEEE/ACM DAC, pp. 370-375, 2009.

[5] D.Tannir, R. Khazaka , "Efficient Nonlinear Distortion Analysis of RF Circuits," Proc. Design Autom. Test Europe Conf., pp. 1-6, 2007.

[6] S. Akatimagool, "Fast Iterative Method Package for High Frequency Circuits Analysis,'IEEE International Symposium on Circuits and Systems -ISCAS.,pp. 5970-5973, November 2005.

[7] E. Richalot, M.F. Wong, H. Baudrand, V. Fouad-Hanna, "an iterative method for modelling of antennas," IEEE APS., pp. 194-200,Jan. 2001.

[8] M. Tellach,Y. Lamhene ,B.Haraoubia ,H.Baudrand , “ An numerical method based iterative process to characterize microwave planar circuits, "International Journal of Computing, vol.7, pp. 86-94, Augest 2014.

[9] H. Megnafi, N. Boukli hacene, "An Efficient Analysis of Microstrip Trisection Filters Using an Iterative Method Combined by Approach 
Multi-Scale," International Journal of Electromagnetics and Applications, Vol. 3, No. 2, pp. 9-14, 2013.

[10] H. Megnafi, N. Boukli hacene, "Analysis of Microstrip Cascaded Quadruplet Filters by a Multi-Scale Wave Concept Iterative Procedure (M.WCIP)," Software Engineering, Vol. 2, No. 4, pp.101-105, 2012.

[11] H. Megnafi, N. Boukli hacene, N. Raveu, H. Baudrand, "A Monolayer Passive Structure Analysis by WCIP method,"6th International Conference on Sciences of Electronics, pp.212-216, 2012.

[12] D. Bajon, H. Baudrand, "Application of wave concept iterative procedure (WCIP) to planar circuits,"Microtec'2000, pp. 864-868, 2000.

[13] H. Trabelsi, A. Gharsallah,H. Baudrand,"Analysis of Microwave Circuits Including Lumped Elements Based on the Iterative Method," Inc. Int J RF and Microwave CAE, Vol 13., pp 269-275, 2003.

[14] Nuangpirom, P., Inchan, S. Akatimagool, "Wave Iterative Method for Patch Antenna Analysis," Applied Mathematics, vol 6., pp. 403413,2015 .

[15] S. El rabaie, vincent, f.fusco, c. Stewart, "Harmonic balance evaluation of nolinear microwave circuit-A tutorial approch,'I.E.E.E. Transactions on Education, vol. 31, pp. 181-192, 1988.
[16] M. S. Nakhla, J. Vlach, "A Piecewise Harmonic Balance Technique for Determination of Periodic Response of Nonlinear Systems, " IEEE Trans. Circuits and Systems, Vol. 23, No.2, pp. 85-91, Feb. 1976.

[17] R. J. Gilmore, M. B. Steer, "Nonlinear Circuit Analysis Using the Method of Harmonic Balance-A Review of the Art. Part I. Introductory Concepts," International journal of Microwave and Millimeter-Wave Computer-Aided Engineering, Vol. 1, No. 1, pp. 22 37, 1991.

[18] J. E. Rayas-Sanchez, F. Lara-Rojo, E. MArtcinez-Guerrero, "A linear inverse space mapping (lism) algorithm to design linear and nonlinear RF and microwave circuit, "IEEE Trans. Microw. Theory Tech., vol. 53, no. 3, pp. 960-968, Mar. 2005.

[19] L.Hans-Dieter, Z.Xingqi,"The Harmonic Balance Method", ECE 1254 project report, Spring 2013.

[20] S. M. Winter, H. J. Ehm, A. Koelpin, and R. Weigel , "Diode Power Detector DC Operating Point in Six-Port Communications," Proc. European Microwave Conf, pp. 795-798, 2007. 